\title{
Redshift and gauge choice
}

\author{
Harald Skarke* \\ Institut für Theoretische Physik, Technische Universität Wien \\ Wiedner Hauptstraße 8-10, 1040 Wien, Austria
}

\begin{abstract}
We show that a specific gauge choice comes extremely close to defining a frame whose preferred observers see a dipole-free CMB. In this gauge the metric is the product of a scale factor depending on all spacetime coordinates, and a metric featuring an expansion-free geodesic timelike vector field. This setup facilitates the computation of redshift and other distance measures and explains why we can have a highly isotropic CMB despite large inhomogeneities.
\end{abstract}

\footnotetext{
*e-mail: skarke@hep.itp.tuwien.ac.at
} 


\section{Introduction}

The almost perfect isotropy of the cosmic microwave background (CMB) is among the pillars of the cosmological standard model according to which our universe can be described, at large scales, as a Friedmann-Lemaitre-Robertson-Walker (FLRW) universe with small perturbations. This isotropy comes at different levels (see [1] for CMB data from Planck and [2] for the peculiar velocities, or [3] for a useful summary): the actual observations (terrestrial or from satellites) show deviations in temperature of $\delta T / T \approx 0.12 \%$, but once the dipole contribution is subtracted, this improves to a value of $\delta_{\mathrm{df}} \approx 10^{-5}$ (here and in the following, we abbreviate $\delta T / T$ by $\delta$ and use subscripts such as 'df' for 'dipole-free' to indicate which observer we are referring to). This means that an observer passing through our solar system at a velocity of $370 \mathrm{~km} / \mathrm{sec}$ (in the right direction) will see the latter spectacularly small level; on the other hand, an observer comoving with our local galaxy group sees an anisotropy of $\delta_{\lg } \approx 0.2 \%$.

According to the Copernican principle, the situation should be similar at most locations in the present era. It is important to note the difference between $\delta_{\mathrm{df}}$ and $\delta_{\mathrm{lg}}$, not only in size $\left(\delta_{\mathrm{df}} \approx 10^{-5} \ll \delta_{\mathrm{lg}} \approx 2 \times 10^{-3}\right)$, but also in quality: whereas $\delta_{\mathrm{df}}$ is determined by a full celestial sphere's worth of observations, the value of $\delta_{\mathrm{lg}}$ comes from a single draw from a distribution with mean zero. For these reasons, we would very much prefer the use of $\delta_{\mathrm{df}}$ over that of $\delta_{\mathrm{lg}}$ in an analysis of the structure of the universe. In other words, we want to work in a frame comoving with the CMB, not with the matter.

The wavelength of a CMB photon is the product of its value at last scattering and the redshift factor picked up on the way to the observer. Unless one believes in strange nonlocal correlations between the two, one can only conclude that neither the original wavelength nor the redshift factor should feature deviations that are larger than the ones seen by the observer. In the present work we will be interested only in the extremely precise matching of the redshifts in the different directions.

The celebrated Ehlers-Geren-Sachs (EGS) theorem [4] states that the existence of a perfectly isotropic radiation background combined with reasonable assumptions on the matter content of the universe implies FLRW. There is a number of generalizations to 'almost EGS' theorems (e.g. $[5,6,7,8])$ stating that small deviations from isotropy should lead only to small deviations from FLRW; see section 11.1 of Ref. [9] for a very clear summary. These works usually (with an exception in [7]) assume that the radiation 4-velocity (i.e. the velocity field $u_{\mathrm{df}}$ of the dipole-free 
observers) is geodesic. This is an additional input which can be argued for only if one does not distinguish the CMB frame from the matter frame. Thus it holds only at the level of $\delta_{\mathrm{lg}}$, not at the level of $\delta_{\mathrm{df}}$.

In the present work we are interested in precision at the level of $\delta_{\mathrm{df}} \approx 10^{-5}$, so we do not take the radiation velocity to be geodesic. Our analysis will rely on redshift rather than distribution functions for the radiation, which simplifies matters considerably. The timelike vector field $u_{\mathrm{df}}$ that determines a preferred observer at every spacetime point can, in principle, be completed to an orthonormal frame $\left\{e_{0}=u_{\mathrm{df}}, e_{1}, e_{2}, e_{3}\right\}$ which we would call a CMB frame. In practice the requirement of a vanishing dipole is highly nonlocal and therefore analytically intractable. Instead, we are going to work with a locally well-defined quantity which, as we shall explicitly verify, comes very close to defining the level of anisotropy. It turns out that this quantity can be simplified by a conformal transformation, and that the most important contributions to it can be eliminated by a gauge choice. The physical observable $\delta_{\mathrm{df}}$ is of course gauge invariant and can therefore be computed in any gauge. Choosing the one suggested here makes it particularly transparent why $\delta_{\mathrm{df}}$ is so small despite the fact that the actual universe shows a considerable amount of inhomogeneity. Working in this gauge significantly improves the tractability of light propagation compared to the synchronous and the longitudinal gauge, which are the ones that are used most frequently. An explicit comparison in linear perturbation theory shows that the metric perturbations in the new gauge are not much larger than those in the longitudinal gauge, which is usually considered to be optimal in that respect.

In the next section we introduce a quantity that vanishes if an isotropically redshifted CMB is observed everywhere, and show how it simplifies under a conformal transformation. In section 3 we formulate a gauge that eliminates two of three contributions to this quantity and thereby comes close to defining a CMB frame; we also give explicit conditions on a metric implementing this gauge. Section 4 contains an analysis of this metric in linear perturbation theory and comparisons with other gauges. In the final section we argue that other distance measures are also well behaved in the new gauge, make some remarks on the controversy about the impact of inhomogeneities on the expansion of the universe, and discuss open questions about our gauge. 


\section{Redshift and conformal transformation}

We consider a photon emitted at some point $x_{e}$ by a source moving along a worldline with a tangent vector $u_{e}$ normalized to $u_{e}^{2}=g_{\mu \nu} u_{e}^{\mu} u_{e}^{\nu}=-1$, where $g_{\mu \nu}$ is the pseudo-Riemannian spacetime metric of type -+++ . This photon propagates along a lightlike geodesic which we describe by an affine parameter $\lambda$ such that the tangent vector to the geodesic is $k^{\mu}=d x^{\mu} / d \lambda$. The redshift $z_{e \rightarrow o}$, as seen by an obvserver at $x_{o}$ whose wordline has the tangent vector $u_{o}$ (normalized to $u_{o}^{2}=-1$ ), is determined by the well-known formula

$$
1+z_{e \rightarrow o}=\frac{(u \cdot k)_{e}}{(u \cdot k)_{o}}
$$

In an idealized universe in which every spacetime point admits a distinguished observer who sees a perfectly isotropically redshifted last scattering surface, there would exist a global vector field $u$ characterizing such observers, as well as a globally well defined function

$$
a(x)=1+z_{\mathrm{lss} \rightarrow x}=\frac{(u \cdot k)_{\mathrm{lss}}}{(u \cdot k)_{x}}
$$

that determines this redshift. We could then determine the redshifts between preferred observers via

$$
1+z_{e \rightarrow o}=\frac{a\left(x_{o}\right)}{a\left(x_{e}\right)}
$$

as a direct consequence of Eqs. (1) and (2). Along any geodesic described with an affine parameter $\lambda$ and tangent vector $k$, the value of $a(x)(u \cdot k)(x)$ would remain constant and therefore the quantity

$$
d(x, k)=\frac{d}{d \lambda}[a(x)(u \cdot k)(x)]
$$

would have to vanish at every spacetime point $x$ for every lightlike tangent vector $k$ at $x$.

For an arbitrary timelike vector field $u$ and non-vanishing scalar $a$, where $d(x, k)$ need not vanish, a redshift formula can still be obtained by noting that

$$
\ln [-a(x)(u \cdot k)(x)]_{e}^{o}=\int_{e}^{o} \frac{d(x, k)}{a(x)(u \cdot k)(x)} d \lambda
$$

implies

$$
1+z_{e \rightarrow o}=\frac{(u \cdot k)_{e}}{(u \cdot k)_{o}}=\frac{a\left(x_{o}\right)}{a\left(x_{e}\right)} \exp \left(-\int_{e}^{o} \frac{d(x, k)}{a(x)\left(u_{\rho} k^{\rho}\right)(x)} d \lambda\right)
$$


In the following we would like to treat the requirement

$$
\langle d(x, k)\rangle=0, \quad\left\langle d(x, k)^{2}\right\rangle \text { small, }
$$

where $\langle\cdots\rangle$ should represent the average over the celestial sphere,

$$
\langle\cdots\rangle=\frac{1}{4 \pi} \int \cdots d \Omega
$$

as a local proxy for the conditions defining the CMB frame. Using the facts that differentiation by $\lambda$ corresponds to covariant differentiation along $k$ and that $k^{\nu} k_{\mu ; \nu}=0$ we get

$$
d(x, k)=k^{\nu}[a(x)(u \cdot k)(x)]_{, \nu}=a_{, \nu} k^{\nu}(u \cdot k)+a u_{\mu ; \nu} k^{\nu} k^{\mu} .
$$

Motivated by the FLRW case, we introduce the conformally transformed quantities

$$
\hat{g}_{\mu \nu}=a^{-2} g_{\mu \nu}, \quad \hat{u}_{\mu}=a^{-1} u_{\mu}, \quad \hat{u}^{\mu}=\hat{g}^{\mu \nu} \hat{u}_{\nu}=a u^{\mu}
$$

with $\hat{u}_{\mu} \hat{u}_{\nu} \hat{g}^{\mu \nu}=u_{\mu} u_{\nu} g^{\mu \nu}=-1$. Then a short calculation gives

$$
a^{2} \hat{u}_{\mu \nmid \nu}=a u_{\mu ; \nu}+a_{, \mu} u_{\nu}-a_{, \rho} u^{\rho} g_{\mu \nu}
$$

where $\hat{j}$ denotes covariant differentiation with respect to $\hat{g}$. Contraction with $k^{\mu} k^{\nu}$ shows that

$$
d(x, k)=\Delta_{\mu \nu}(x) k^{\mu} k^{\nu}=\hat{\Delta}_{\mu \nu}(x) \hat{k}^{\mu} \hat{k}^{\nu}
$$

with

$$
\Delta_{\mu \nu}=a u_{(\mu ; \nu)}+a_{,(\mu} u_{\nu)}-a_{, \rho} u^{\rho} g_{\mu \nu}=a^{2} \hat{\Delta}_{\mu \nu}, \quad \hat{\Delta}_{\mu \nu}=\hat{u}_{(\mu ; \nu)} .
$$

Thus Killing's equation $\hat{u}_{(\mu \hat{;} \nu)}=0$ implies $d(x, k)=0$, and with a little work the converse can also be shown. This corresponds to the well-known result [10] that a spacetime admits a perfectly isotropic CMB background if and only if its metric is conformal to a metric with a timelike Killing vector; this fact is essential for the derivation of the EGS theorem [4].

The standard decomposition (see e.g. chapter 4 of [9]) of

$$
g_{\mu \nu}=-u_{\mu} u_{\nu}+h_{\mu \nu}
$$

into projection operators $-u_{\mu} u_{\nu}$ (timelike) and $h_{\mu \nu}$ (spacelike), with $u^{\mu} h_{\mu \nu}=0$ and $h^{\mu \nu} h_{\mu \nu}=3$, (or, equivalently, $\hat{g}_{\mu \nu}=-\hat{u}_{\mu} \hat{u}_{\nu}+\hat{h}_{\mu \nu}$ etc.) affords a decomposition of any symmetric tensor $\Delta_{\mu \nu}$ as

$$
\Delta_{\mu \nu}=u_{\mu} u_{\nu} \Delta^{\mathrm{St}}+h_{\mu \nu} \Delta^{\mathrm{Ss}}-u_{\mu} \Delta_{\nu}^{\mathrm{V}}-u_{\nu} \Delta_{\mu}^{\mathrm{V}}+\Delta_{\mu \nu}^{\mathrm{T}}
$$


in terms of scalars $\Delta^{\mathrm{St}}$ and $\Delta^{\mathrm{Ss}}$ (related to the time and space projections, respectively), a vector $\Delta_{\mu}^{\mathrm{V}}$ satisfying $\Delta_{\mu}^{\mathrm{V}} u^{\mu}=0$ and a symmetric tensor $\Delta_{\mu \nu}^{\mathrm{T}}$ satisfying $\Delta_{\mu \nu}^{\mathrm{T}} u^{\mu}=0$ and $\Delta_{\mu \nu}^{\mathrm{T}} h^{\mu \nu}=0$.

Assuming that we have parametrized the geodesic in such a way that $u \cdot k=-1$ at the point $x$ where we compute $d(x, k)$, writing

$$
k^{\mu}=u^{\mu}+e^{\mu}
$$

and using the conditions $u^{2}=-1$ and $k^{2}=0$, we find that

$$
u \cdot e=0, \quad e^{2}=1 \quad \text { and } \quad e^{\mu} h_{\mu \nu}=e_{\nu}
$$

i.e. $e$ must be a spacelike unit vector orthogonal to $u$. Applying this to Eq. (12) with the decomposition (15), we find

$$
d(x, k)=\Delta^{\mathrm{S}}+2 \Delta_{\nu}^{\mathrm{V}} e^{\nu}+\Delta_{\mu \nu}^{\mathrm{T}} e^{\mu} e^{\nu} \quad \text { with } \quad \Delta^{\mathrm{S}}=\Delta^{\mathrm{St}}+\Delta^{\mathrm{Ss}}
$$

In order to evaluate averages of the type (8) we introduce spacelike unit vectors $e_{1}^{\mu}, e_{2}^{\mu}, e_{3}^{\mu}$ that form a tetrad together with $u^{\mu}$, and define $e^{\mu}(\Omega)=\cos \varphi \sin \vartheta e_{1}^{\mu}+\ldots$ through standard spherical coordinates $\Omega=(\varphi, \vartheta)$; these quantities satisfy

$$
\left\langle e^{\mu_{1}} \cdots e^{\mu_{2 p+1}}\right\rangle=0, \quad\left\langle e^{\mu} e^{\nu}\right\rangle=\frac{1}{3} h^{\mu \nu}, \quad\left\langle e^{\mu} e^{\nu} e^{\rho} e^{\sigma}\right\rangle=\frac{1}{15}\left(h^{\mu \nu} h^{\rho \sigma}+h^{\mu \rho} h^{\nu \sigma}+h^{\mu \sigma} h^{\nu \rho}\right) .
$$

Note how Eq. (12) expresses the quantity $d(x, k)$, which depends both on the spacetime coordinates $x^{\mu}$ and the tangent space coordinates $k^{\mu}$, in terms of the tensor quantity $\Delta_{\mu \nu}$ (depending only on the $x^{\mu}$ ) and the bilinear $k^{\mu} k^{\nu}$. Therefore $\Delta^{\mathrm{S}}, \Delta_{\nu}^{\mathrm{V}}$ and $\Delta_{\mu \nu}^{\mathrm{T}}$ do not depend on $e^{\mu}$, and one can directly apply (19) to find

$$
\langle d(x, k)\rangle=\Delta^{\mathrm{S}}, \quad\left\langle d(x, k)^{2}\right\rangle=\left(\Delta^{\mathrm{S}}\right)^{2}+\frac{4}{3} h^{\mu \nu} \Delta_{\mu}^{\mathrm{V}} \Delta_{\nu}^{\mathrm{V}}+\frac{2}{15} \Delta_{\mu \nu}^{\mathrm{T}} h^{\nu \rho} \Delta_{\rho \sigma}^{\mathrm{T}} h^{\sigma \mu} .
$$

Returning to the specific form (13) of $\Delta_{\mu \nu}$, application of the projection operators (in the 'hatted' version) gives $\hat{\Delta}^{\mathrm{St}}=0$ (so that $\left.\hat{\Delta}^{\mathrm{S}}=\hat{\Delta}^{\mathrm{Ss}}\right)$ and

$$
\begin{aligned}
\hat{\Delta}^{\mathrm{S}} & =\frac{1}{3} \hat{g}^{\mu \nu} \hat{u}_{\mu \hat{i}, \nu} \\
\hat{\Delta}_{\mu}^{\mathrm{V}} & =\frac{1}{2} \hat{u}_{\mu \hat{\rho} \rho} \hat{u}^{\rho}, \\
\hat{\Delta}_{\mu \nu}^{\mathrm{T}} & =\hat{u}_{(\mu \hat{\mu} \nu)}-\hat{h}_{\mu \nu} \hat{\Delta}^{\mathrm{S}}+\hat{u}_{\mu} \hat{\Delta}_{\nu}^{\mathrm{V}}+\hat{u}_{\nu} \hat{\Delta}_{\mu}^{\mathrm{V}},
\end{aligned}
$$


i.e. these quantities correspond to the expansion, the acceleration and the shear of the timelike vector field $\hat{u}$ with respect to the metric $\hat{g}$.

This has the following effects on the redshift. In the integral in Eq. (6) we can write $\left(\hat{\Delta}_{\mu \nu} / \hat{u}_{\rho}\right) k^{\mu} k^{\nu}$ instead of $d(x, k) /\left(a u_{\rho}\right)$. Furthermore, since $\left(k^{\mu} k^{\nu} / k^{\rho}\right) d \lambda$ is invariant under arbitrary reparametrizations of the geodesic, we can replace it by $\left(\tilde{k}^{\mu} \tilde{k}^{\nu} / \tilde{k}^{\rho}\right) d \tilde{\lambda}$ with $\tilde{k}^{\mu}=\hat{u}^{\mu}+\hat{e}^{\mu}$ chosen such that $\hat{u}_{\rho} \tilde{k}^{\rho}=-1$ everywhere along the geodesic; the factor $\hat{\Delta}_{\mu \nu}$ is unaffected because it depends only on $x$, not on $k$. Thus the argument of the exponential in Eq. (6) becomes $\int_{e}^{o} \hat{\Delta}_{\mu \nu} \tilde{k}^{\mu} \tilde{k}^{\nu} d \tilde{\lambda}$. Then, using the analog of Eq. (18) for the metric $\hat{g}$, we get

$$
1+z_{e \rightarrow o}=\frac{a\left(x_{o}\right)}{a\left(x_{e}\right)} \exp \left(\int_{e}^{o}\left(\hat{\Delta}^{\mathrm{S}}+2 \hat{\Delta}_{\nu}^{\mathrm{V}} \hat{e}^{\nu}+\hat{\Delta}_{\mu \nu}^{\mathrm{T}} \hat{e}^{\mu} \hat{e}^{\nu}\right) d \tilde{\lambda}\right)
$$

for our preferred sources and observers whose worldlines have tangent vectors $u^{\mu}$. If the actual emitter ('ae') and actual observer ('ao') have different tangent vectors (but the same positions), we must of course correct this via

$$
1+z_{a e \rightarrow a o}=\left(1+z_{a e \rightarrow e}\right)\left(1+z_{e \rightarrow o}\right)\left(1+z_{o \rightarrow a o}\right)
$$

where $1+z_{a e \rightarrow e}$ and $1+z_{o \rightarrow a o}$ are just the standard special-relativistic Doppler factors coming from the relative velocities between the actual and preferred sources and observers, respectively.

\section{Gauge choice and metric}

The actual universe features deviations from homogeneity, so we do not expect all components of $\Delta_{\mu \nu}$ to vanish. Why can we nevertheless find a local frame in which the CMB has almost exactly the same temperature in all directions? We propose that this can be explained in the following manner. Eqs. (24), (25) give the correct redshift for arbitrary sources and observers and arbitrary functions $a(x)$ and vector fields $u(x)$. The result is of course independent of the choice of $a$ and $u$; for most choices, several of the factors occurring in Eqs. (24), (25) will get large or small, and the computation of the CMB redshift will involve cancellations between these factors. If, however, we choose our setup such that $a(x)$ varies very little on the last scattering surface, the relative velocities of the CMB sources are very small, and the observer is the preferred one, then the only factor that can still exhibit a strong direction dependence is the exponential occurring in Eq. (24). If we want to interpret the average of $a\left(x_{o}\right) / a\left(x_{e}\right)$, with 
the source positions $x_{e}$ on the last scattering surface, as 'the' redshift, and every other factor as providing at most a further small fluctuation, we need to ensure that the integral in Eq. (24) is small. We suggest to achieve this by choosing $a$ and $u$ in such a way that

$$
\Delta^{\mathrm{S}}=0, \quad \Delta_{\mu}^{\mathrm{V}}=0
$$

which is an admissible gauge choice. Indeed, $\Delta^{\mathrm{S}}$ and $\Delta_{\mu}^{\mathrm{V}}$ correspond to $1+3=4$ degrees of freedom, which is just the number of quantities that can be fixed by a gauge. This choice reduces the redshift formula (24) to

$$
1+z_{e \rightarrow o}=\frac{a\left(x_{o}\right)}{a\left(x_{e}\right)} \exp \left(\int_{e}^{o} \hat{\Delta}_{\mu \nu}^{\mathrm{T}} \hat{e}^{\mu} \hat{e}^{\nu} d \tilde{\lambda}\right) .
$$

The tracelessness of $\hat{\Delta}_{\mu \nu}^{\mathrm{T}}$ together with statistical isotropy ensures that the integrand $\hat{\Delta}_{\mu \nu}^{\mathrm{T}} \hat{e}^{\mu} \hat{e}^{\nu}$ has vanishing expectation value, and in the next section we shall also see that it vanishes in linear perturbation theory. Thus it is not so surprising that the integral is small.

In terms of the original timelike field $u$, the effects of this choice on the expansion $\Theta=$ $h^{\mu \nu} u_{\mu ; \nu}$, the acceleration $\dot{u}_{\mu}=u_{\mu ; \rho} u^{\rho}$, the shear $\sigma_{\mu \nu}=u_{\mu ; \nu}^{\mathrm{PSTF}}$ (the projected symmetric tracefree part of $u_{\mu ; \nu}$, i.e. what remains after symmetrizing, projecting with $h$ and removing the $h$-trace) and the vorticity $\omega_{\mu \nu}=h_{\mu}{ }^{\rho} h_{\nu}{ }^{\sigma} u_{[\rho ; \sigma]}$ are easily found with the help of Eq. (11):

$$
\dot{u}_{\mu}=h_{\mu}{ }^{\nu} \frac{a_{, \nu}}{a}, \quad \Theta=3 u^{\rho} \frac{a_{, \rho}}{a}, \quad \sigma_{\mu \nu}=a \hat{u}_{(\mu \hat{\mu})}, \quad \omega_{\mu \nu}=a \hat{u}_{[\mu \hat{;} \nu]} .
$$

In words, expansion and acceleration correspond to the timelike and spacelike components of $(\ln a)_{, \mu}$, respectively; shear and vorticity are multiples of the corrresponding quantities in the conformally transformed frame.

Let us now find explicit coordinates that implement our gauge (26). Choosing $\hat{u}$ to be the vector with components $\hat{u}^{0}=1$ and $\hat{u}^{i}=0$, we get $\hat{g}_{00}=-1, \hat{u}_{\hat{;} \rho}^{\mu}=\hat{u}_{, \rho}^{\mu}+\hat{\Gamma}^{\mu}{ }_{\rho \nu} \hat{u}^{\nu}=\hat{\Gamma}_{\rho 0}^{\mu}$ and therefore

$$
\hat{u}_{\mu \hat{j} \rho}=\hat{\Gamma}_{\mu \rho 0} .
$$

Upon demanding $0=2 \hat{\Delta}_{\mu}^{\mathrm{V}}=\hat{u}_{\mu \hat{\rho} \rho} \hat{u}^{\rho}=\hat{\Gamma}_{\mu 00}=\hat{g}_{\mu 0,0}$, the metric takes the form $d s^{2}=a^{2} d \hat{s}^{2}$ with

$$
d \hat{s}^{2}=-\left(d x^{0}-V_{i} d x^{i}\right)^{2}+\gamma_{i j} d x^{i} d x^{j}
$$

where $a$ and $\gamma_{i j}$ can depend on all coordinates $x^{\mu}$ whereas $V_{i}$ depends only on the spatial coordinates $x^{j}$. The inverse metric $\hat{g}^{\mu \nu}$ has the components

$$
\hat{g}^{00}=-1+V_{i} \gamma^{i j} V_{j}, \quad \hat{g}^{0 j}=\gamma^{j k} V_{k}, \quad \hat{g}^{i j}=\gamma^{i j},
$$


where $\gamma^{i j}$ is defined by the requirement $\gamma^{i j} \gamma_{j k}=\delta_{k}^{i}$. In matrix notation, the original metric and its inverse are given by

$$
g=a^{2}\left(\begin{array}{cc}
-1 & V^{T} \\
V & \gamma-V V^{T}
\end{array}\right), \quad g^{-1}=a^{-2}\left(\begin{array}{cc}
-1+V^{T} \gamma^{-1} V & V^{T} \gamma^{-1} \\
\gamma^{-1} V & \gamma^{-1}
\end{array}\right) .
$$

Finally, $0=6 \hat{\Delta}^{\mathrm{S}}=2 \hat{g}^{\mu \nu} \hat{u}_{\mu \hat{\mu}, \nu}=2 \hat{g}^{\mu \nu} \hat{\Gamma}_{\mu \nu 0}=\hat{g}^{\mu \nu}\left(\hat{g}_{\mu \nu, 0}+\hat{g}_{\mu 0, \nu}-\hat{g}_{\nu 0, \mu}\right)=\hat{g}^{\mu \nu} \hat{g}_{\mu \nu, 0}=\hat{g}^{i j} \gamma_{i j, 0}=$ $\operatorname{tr}\left(\gamma^{-1} \gamma_{, 0}\right)=(\operatorname{tr} \ln \gamma)_{, 0}=(\ln \operatorname{det} \gamma)_{, 0}$ implies $x^{0}$-independence of $\operatorname{det} \gamma$.

The conditions $V_{i, 0}=0$ and $(\operatorname{det} \gamma)_{, 0}=0$ do not completely fix the form of our metric (30). For example, they also hold in a transformed frame $\left\{\tilde{x}^{\mu}\right\}$ with

$$
\tilde{x}^{0}=x^{0}+f\left(x^{j}\right), \quad \tilde{x}^{i}=\tilde{x}^{i}\left(x^{j}\right) .
$$

We can use parts of this freedom to assign a single time coordinate to the initial singularity and to set $\operatorname{det} \gamma=1$.

\section{Linear perturbation theory}

We would now like to consider the consequences of our gauge choice (26) in the context of linear perturbation theory [11]. Our notation will be similar to that of Refs. [12, 9] which we also recommend for further details. A metric corresponding to a small perturbation of the conformally flat case is given, before gauge fixing, by

$$
d s^{2}=a_{\mathrm{h}}^{2}\left(x^{0}\right)\left\{-(1+2 \phi)\left(d x^{0}\right)^{2}+2\left(B_{, i}-S_{i}\right) d x^{i} d x^{0}+\left[(1-2 \psi) \delta_{i j}+2 E_{, i j}+2 F_{(i, j)}+h_{i j}\right] d x^{i} d x^{j}\right\} ;
$$

here $a_{\mathrm{h}}\left(x^{0}\right)$ represents the scale factor for the corresponding homogeneous case $\left(g_{\mathrm{h}}\right)_{\mu \nu}=a_{\mathrm{h}}^{2} \eta_{\mu \nu}$; $\phi, \psi, B$ and $E$ are scalars; $S_{i}$ and $F_{i}$ are transverse vectors (i.e. they satisfy $\delta^{i j} S_{i, j}=0$ and $\left.\delta^{i j} F_{i, j}=0\right) ; h_{i j}$ is a symmetric traceless transverse tensor $\left(h_{i j}=h_{j i}, \delta^{i j} h_{i j}=0, \delta^{i k} h_{i j, k}=0\right)$. The gauge freedom $x^{\mu} \rightarrow \tilde{x}^{\mu}\left(x^{\nu}\right)$ can be expressed at the linearized level in terms of a transverse vector $\xi^{i}$ and scalars $\xi^{0}$ and $\xi$; the corresponding transformations

$$
\begin{gathered}
\tilde{\phi}=\phi-\frac{a_{\mathrm{h}}^{\prime}}{a_{\mathrm{h}}} \xi^{0}-\xi_{, 0}^{0}, \quad \tilde{\psi}=\psi+\frac{a_{\mathrm{h}}^{\prime}}{a_{\mathrm{h}}} \xi^{0}, \quad \tilde{B}=B+\xi^{0}-\xi_{, 0}, \quad \tilde{E}=E-\xi, \\
\tilde{F}_{i}=F_{i}-\xi_{i}, \quad \tilde{S}_{i}=S_{i}+\xi_{i, 0}, \quad \tilde{h}_{i j}=h_{i j}
\end{gathered}
$$

can then be used to eliminate two of the scalars and one of the transverse vectors. The two most popular gauge choices are longitudinal gauge with $B=E=0$ (usually accompanied 
by neglecting vector and tensor modes), and synchronous gauge, which manifests itself at the linearized level as $\phi=B=0, S_{i}=0$.

A well-known solution to the Einstein equations for irrotational dust with $\Lambda=0$ (hence $a_{\mathrm{h}}=$ const $\left.\times\left(x^{0}\right)^{2}\right)$, which is believed to give a good description of the early matter dominated era of our universe, relies on a single time-independent function $\phi_{\mathrm{N}}$ which is just the Newtonian potential. In the longitudinal gauge this solution is given by $\phi_{\text {long }}=\psi_{\text {long }}=\phi_{\mathrm{N}}$; it can be transformed to the synchronous gauge via $\xi^{0}=x^{0} \phi_{\mathrm{N}} / 3, \xi=\left(x^{0}\right)^{2} \phi_{\mathrm{N}} / 6$, resulting in $E_{\mathrm{sync}}=$ $-(1 / 6)\left(x^{0}\right)^{2} \phi_{\mathrm{N}}, \psi_{\text {sync }}=(5 / 3) \phi_{\mathrm{N}}$. In the latter case, second derivatives of $\phi_{\mathrm{N}}$ occur in the metric and tend to make the perturbations large for moderate $x^{0}$, which is often used as an argument against employing the synchronous gauge in situations other than the very early universe.

What about the gauge (26) and the corresponding metric (32)? If we assume that we have used some of our residual gauge freedom to set $\operatorname{det} \gamma=1$, then in the linearized version $\gamma_{i j}-\delta_{i j}$ must be traceless. Writing $a=(1+\phi) a_{\mathrm{h}}$, this implies $\delta^{i j} E_{, i j}=3(\phi+\psi)$. It turns out that without violating our gauge conditions we can set $B$ and $S_{i}$ to zero, so that the metric becomes (up to quadratic and higher terms)

$$
d s^{2}=a_{\mathrm{h}}^{2}\left(x^{0}\right)(1+2 \phi)\left\{-\left(d x^{0}\right)^{2}+\left[\delta_{i j}+2\left(E_{, i j}-\frac{1}{3} \delta^{k l} E_{, k l} \delta_{i j}\right)+2 F_{(i, j)}+h_{i j}\right] d x^{i} d x^{j}\right\} .
$$

For the special solution considered above we can get to this form by applying a transformation with $\xi^{0}=0, \xi^{i}=0$ and $\xi$ satisfying $\xi_{, 0}=0$ and $\delta^{i j} \xi_{, i j}=-6 \phi_{\mathrm{N}}$ to the metric in the longitudinal gauge. This results in $\phi=\phi_{\mathrm{N}}$ and $E$ chosen such that $\delta^{i j} E_{, i j}=6 \phi_{\mathrm{N}}$. Thus we can interpret $E$ as a gravitational prepotential. In particular, the expressions $E_{, i j}$ occurring in the metric should be roughly of the same order of magnitude as $\phi_{\mathrm{N}}$.

It is instructive to apply our formalism to the metric (34) that is not restricted by a gauge choice. Considering the preferred observer to be the comoving one, we get $a(x)=a_{\mathrm{h}}\left(x^{0}\right)(1+\phi)$ and

$$
d \hat{s}^{2}=-\left(d x^{0}\right)^{2}+2\left(B_{, i}-S_{i}\right) d x^{i} d x^{0}+\left[(1-2 \psi-2 \phi) \delta_{i j}+2 E_{, i j}+2 F_{(i, j)}+h_{i j}\right] d x^{i} d x^{j} .
$$

Using Eq. (29), we find $\hat{\Delta}_{\mu \nu}=\hat{u}_{(\mu \hat{j} \nu)}=\hat{\Gamma}_{(\mu \nu) 0}=\frac{1}{2} \hat{g}_{\mu \nu, 0}$ for a general $\hat{g}_{\mu \nu}$. It is straightforward to compute and decompose this expression for the metric (38), resulting in

$$
\begin{aligned}
\hat{\Delta}^{\mathrm{S}} & =-\psi_{, 0}-\phi_{, 0}+\frac{1}{3} \delta^{i j} E_{, i j 0} \\
\hat{\Delta}_{i}^{\mathrm{V}} & =\frac{1}{2}\left(B_{, i}-S_{i}\right)_{, 0}
\end{aligned}
$$




$$
\hat{\Delta}_{i j}^{\mathrm{T}}=E_{, i j 0}-\frac{1}{3} \delta_{i j} \delta^{k l} E_{, k l 0}+F_{(i, j) 0}+\frac{1}{2} h_{i j, 0} .
$$

We see again how the metric (32) ensures the vanishing of $\Delta^{\mathrm{S}}$ and $\Delta^{\mathrm{V}}$. Expanding Eqs. (24), (25), with source and observer velocities of $v_{e}^{i}$ and $v_{o}^{i}$, respectively, to the linear level, results in $1+z_{a e \rightarrow a o}=\frac{a_{\mathrm{h}}\left(x_{o}\right)}{a_{\mathrm{h}}\left(x_{e}\right)}\left\{1+\left[\phi+v_{i} \hat{e}^{i}\right]_{e}^{o}+\int_{e}^{o}\left[-\psi_{, 0}-\phi_{, 0}+\left(B_{, i}-S_{i}\right)_{, 0} \hat{e}^{i}+\left(E_{, i j 0}+F_{(i, j) 0}+\frac{1}{2} h_{i j, 0}\right) \hat{e}^{i} \hat{e}^{j}\right] d \tilde{\lambda}\right\}$.

This expression is in full agreement with corresponding results in the literature. (To get, for example, Eq. (11) of Ref. [13], one has to note several different naming and sign conventions including the directions of the unit vectors, and to partially integrate the $\left(B_{, i}-S_{i}\right)$-term. $)$ As explained in detail in Ref. [13], Eq. (42) contains all the standard contributions to the redshift, such as, for example, the Sachs-Wolfe effect [14].

For the dust solution considered above, neither the longitudinal gauge nor the gauge advocated here lead to corrections at the linearized level since the linearized fields are $x^{0}$-independent in these gauges; in contrast to this, the synchronous gauge features corrections because $E_{\text {sync }}=$ $-(1 / 6)\left(x^{0}\right)^{2} \phi_{\mathrm{N}}$, in consistency with observations which show that the matter frame (the preferred frame in the synchronous gauge) substantially differs from the CMB frame. While linear perturbation theory provides an excellent description of the early universe, nonlinearities do play an important role in later eras, and this is where we expect differences between the gauge (26) and some nonlinearly consistent version of the longitudinal gauge such as the Poisson gauge to manifest themselves.

In the simplified model mentioned above one could compute the source velocities as the matter velocities $v_{i}=T_{0 i} / T_{00}=G_{0 i} / G_{00}$ from the components of the energy-momentum tensor and therefore from the Einstein tensor, but this would neglect the different motions of visible and dark matter. A complete analysis of the CMB fluctuations would include an early, perturbative part in which these and many more details are taken into account; this would include the temperature variations, the actual source velocities taking into account the incomplete alignment of dark and hadronic matter, contributions of the radiation field to the energy-momentum tensor, etc. This can be done with the perturbative version (37) of the metric (32), or by transforming results obtained in any other gauge to the present setup. At a point in the history of the universe where linear perturbation theory is still a good approximation but radiation can already be neglected, one should then hand over to a fully relativistic $\Lambda \mathrm{CDM}$ simulation in the gauge (26). 
Let us briefly summarize the results of this section. The present formalism passes the consistency check of providing the correct linearized redshift formula (42) in a general gauge. Our metric is well behaved: in contrast to the synchronous gauge, the linearized expressions do not exhibit a time dependence that would quickly lead to troubles. The integral occurring in the redshift formula (24), which represents those deviations from the uniform case that cannot be attributed to properties of the sources, vanishes at first order of perturbation theory in a simple matter-only model, both in longitudinal gauge and in the gauge (26), but only in the latter the first two contributions $\hat{\Delta}^{\mathrm{S}}$ and $\hat{\Delta}_{\nu}^{\mathrm{V}} \hat{e}^{\nu}$ vanish at all orders. The remaining quantity $\hat{\Delta}_{\mu \nu}^{\mathrm{T}} e^{\mu} e^{\nu}$ has an expectation value of zero at all orders. These facts make our formalism particularly useful for understanding why we observe almost perfect isotropy of the CMB despite the existence of severe inhomogeneities in the non-linear era.

\section{Concluding remarks}

Observational cosmology relies not only on the redshift, but also on other distance measures such as the angular diameter distance and the luminosity distance. These quantities can be computed via arguments based on fluxes. For known redshift, one can use a comparison between the total number of photons emitted per unit of time in a specific frequency range, and the number of photons, in the appropriately transformed frequency range, arriving in a given area at the observer's location. Because of the non-acceleration and non-expansion of the vector field $\hat{u}$ with resepct to $\hat{g}$, the number of photons arriving per unit of $x^{0}$ (the time coordinate related to $\hat{u}$ ) on a suitable hypothetic screen enveloping the source must be identical with the number of photons emitted during the corresponding $x^{0}$-interval of the same duration (as measured with $\hat{g}$ ). Therefore, on average the photon count with respect to $\hat{g}$ behaves like the photon count in a static universe. Upon proper rescalings of the time and area values with the corresponding powers of $a$ one gets formulas for averaged fluxes that are identical in form with those for a homogeneous universe, but with $a_{\mathrm{h}}$ replaced by $a$. Thus the overall expansion, as inferred from measured redshift-distance relations, is given straightforwardly by the values of $a$ at the sources and at our spacetime position.

There have been suggestions (for a small subset, see e.g. [15, 16, 17, 18, 19, 20]) that the perceived acceleration of the universe's expansion may not be due to a cosmological constant or dark energy, but to some effect stemming from the inhomogeneities of the actual universe. 
This possibility is rejected in papers such as [21,22], giving rise to further rounds of controversy $[23,24]$. One of the main points of $[21,22]$ is an attack on the choice of synchronous gauge on which many attempts to explain the data without $\Lambda$ are based; instead the use of the longitudinal gauge is advocated. From the present work it is clear that neither of these gauges is as directly related to observations as the one presented here in Eq. (26).

This makes a thorough investigation of the properties and consequences of this gauge choice highly desirable. Open questions include the following. What residual gauge freedom is there beyond that indicated in (33)? Is the possibility of setting $\hat{g}_{0 i}=V_{i}$ to zero general or specific to linear perturbation theory? What are the Einstein equations in linear and second order perturbation theory, for collisionless dust and more generally? Can we reproduce arguments along the lines of $[21,22]$ ? What can we say beyond perturbation theory, either by analytic arguments or numerically?

Acknowledgements: It is a pleasure to thank Dominik Schwarz for discussions.

\section{References}

[1] Y. Akrami et al. [Planck], [arXiv:1807.06205 [astro-ph.CO]].

[2] Y. Hoffman, H. M. Courtois and R. B. Tully, Mon. Not. Roy. Astron. Soc. 449, no.4, 4494-4505 (2015) doi:10.1093/mnras/stv615 [arXiv:1503.05422 [astro-ph.CO]].

[3] M. Tanabashi et al. [Particle Data Group], Phys. Rev. D 98, no. 3, 030001 (2018). doi:10.1103/PhysRevD.98.030001

[4] J. Ehlers, P. Geren and R. K. Sachs, J. Math. Phys. 9, 1344 (1968). doi:10.1063/1.1664720

[5] W. R. Stoeger, S.J., R. Maartens and G. F. R. Ellis, Astrophys. J. 443, 1 (1995). doi:10.1086/175496

[6] C. A. Clarkson and R. Barrett, Class. Quant. Grav. 16, 3781 (1999) doi:10.1088/02649381/16/12/302 [gr-qc/9906097].

[7] S. Rasanen, Phys. Rev. D 79, 123522 (2009) doi:10.1103/PhysRevD.79.123522 [arXiv:0903.3013 [astro-ph.CO]]. 
[8] C. Clarkson and R. Maartens, Class. Quant. Grav. 27, 124008 (2010) doi:10.1088/02649381/27/12/124008 [arXiv:1005.2165 [astro-ph.CO]].

[9] G. F. R. Ellis, R. Maartens and M. A. H. MacCallum, "Relativistic Cosmology", Cambridge University Press 2012.

[10] G. E. Tauber and J. W. Weinberg, Phys. Rev. 122, no. 4, 1342 (1961). doi:10.1103/PhysRev.122.1342

[11] J. M. Bardeen, Phys. Rev. D 22, 1882 (1980). doi:10.1103/PhysRevD.22.1882

[12] V. F. Mukhanov, H. A. Feldman and R. H. Brandenberger, Phys. Rept. 215, 203 (1992). doi:10.1016/0370-1573(92)90044-Z

[13] J. Yoo, A. Fitzpatrick and M. Zaldarriaga, Phys. Rev. D 80, 083514 (2009) doi:10.1103/PhysRevD.80.083514 [arXiv:0907.0707 [astro-ph.CO]].

[14] R. Sachs and A. Wolfe, Astrophys. J. 147, 73-90 (1967) doi:10.1007/s10714-007-0448-9

[15] D. J. Schwarz, astro-ph/0209584.

[16] D. L. Wiltshire, New J. Phys. 9, 377 (2007) doi:10.1088/1367-2630/9/10/377 [grqc/0702082].

[17] T. Buchert, Gen. Rel. Grav. 32, 105 (2000) doi:10.1023/A:1001800617177 [gr-qc/9906015].

[18] S. Rasanen, JCAP 0611, 003 (2006) doi:10.1088/1475-7516/2006/11/003 [astro$\mathrm{ph} / 0607626]$.

[19] T. Buchert, Gen. Rel. Grav. 40, 467 (2008) doi:10.1007/s10714-007-0554-8 [arXiv:0707.2153 [gr-qc]].

[20] H. Skarke, Eur. Phys. J. C 77, no. 3, 177 (2017) doi:10.1140/epjc/s10052-017-4743-7 [arXiv:1508.01510 [gr-qc]].

[21] A. Ishibashi and R. M. Wald, Class. Quant. Grav. 23, 235 (2006) doi:10.1088/02649381/23/1/012 [gr-qc/0509108].

[22] S. R. Green and R. M. Wald, Class. Quant. Grav. 31, 234003 (2014) doi:10.1088/02649381/31/23/234003 [arXiv:1407.8084 [gr-qc]]. 
[23] T. Buchert et al., Class. Quant. Grav. 32, 215021 (2015) doi:10.1088/02649381/32/21/215021 [arXiv:1505.07800 [gr-qc]].

[24] S. R. Green and R. M. Wald, arXiv:1506.06452 [gr-qc]. 